\title{
Biomarkers for Locally Advanced Hepatocellular Carcinoma Patients Treated with Liver-Directed Combined Radiotherapy
}

\author{
Seung Yeun Chung ${ }^{a, b}$ Kyoung-Jin Kim ${ }^{a}$ Jinsil Seong ${ }^{a}$ \\ aDepartment of Radiation Oncology, Yonsei Cancer Center, Yonsei University College of Medicine, Seoul, \\ Republic of Korea; ${ }^{b}$ Department of Radiation Oncology, Ajou University School of Medicine, Suwon-si, \\ Republic of Korea
}

\section{Keywords}

Hepatocellular carcinoma $\cdot$ Radiotherapy $\cdot$ Liver-directed combined radiotherapy · Biomarker $\cdot$ Tumor progression

\begin{abstract}
Introduction: In the era of biomarker-driven cancer therapy, robust biomarkers for hepatocellular carcinoma (HCC) have not been well-defined. In this hypothesis-generating study, we investigated biomarkers that can be incorporated to predict treatment outcomes in patients with locally advanced HCC who are administered liver-directed combined radiotherapy (LDCRT). Methods: Ninety-nine patients with HCC who were treated with conventional fractionation LDCRT between July 2016 and October 2018 were enrolled in this prospective single-arm study. Clinical outcomes and possible serum biomarkers, including soluble programmed cell death ligand-1 (sPD-L1), interleukin (IL)-10, IL-6, cell-free DNA (cfDNA), inter-alpha inhibitor $\mathrm{H} 4$, and interferon-gam$\mathrm{ma}$, were analyzed. The primary endpoint was disease progression, and additional endpoints were local failure-free rate, intrahepatic failure-free rate, and lung metastasis-free rate. Results: The median follow-up period was 18.7 months. The 1-year progression-free rate was $38.2 \%$. Increasing base-
\end{abstract}

Karger@karger.com www.karger.com/lic

Karger"

GOPEN ACCESS
C 2022 The Author(s).

Published by S. Karger AG, Basel

This is an Open Access article licensed under the Creative Commons Attribution-NonCommercial-4.0 International License (CC BY-NC) (http://www.karger.com/Services/OpenAccessLicense), applicable to the online version of the article only. Usage and distribution for commercial purposes requires written permission. line sPD-L1 per pg/mL, previous treatment history, protein induced by vitamin $\mathrm{K}$ absence-II $>1,629 \mathrm{mAU} / \mathrm{mL}$, and multiple tumors were the adverse factors for progression based on multivariate analysis. Survival tree analysis revealed three prognostic groups for progression, in which patients with multiple lesions and baseline sPD-L1 $\geq 41.07 \mathrm{pg} / \mathrm{mL}$ showed the worst outcomes. For dynamic changes in biomarker levels, SPD-L1 fold change and cfDNA fold-change values were unfavorable factors for progression. Conclusion: Baseline sPD-L1, sPD-L1 fold change, and cfDNA fold-change values showed the highest potential as biomarkers for predicting post-treatment progression after LDCRT in HCC patients. By incorporating clinical factors, these biomarkers may be useful for devising a biomarker-driven treatment paradigm in locally advanced HCC.

(c) 2022 The Author(s)

Published by S. Karger AG, Basel

\section{Introduction}

Although the survival of patients with hepatocellular carcinoma (HCC) has improved due to new treatment options, HCC prognosis remains dismal [1]. Only a minority of patients are diagnosed at an early stage when curative
Correspondence to:

Jinsil Seong, jsseong@yuhs.ac 
surgery is possible. Although tyrosine kinase inhibitors, or more recently, combinations of tyrosine kinase inhibitors and immune checkpoint inhibitors, are recommended for locally advanced HCC, many other treatment modalities are used in real-world practice [2]. Liver-directed combined radiotherapy (LDCRT) improves oncologic outcomes as a bridge to curative surgery in locally advanced HCC [3]. A substantial proportion of patients can be converted to curative resection, achieving a 5-year survival rate of $>50 \%$, while some patients develop lung metastasis. Considering the extreme heterogeneity among locally advanced HCC, prediction of therapeutic outcomes seems necessary for providing personalized treatment.

Different responses to treatment may occur due to tumor heterogeneity and the host-immune system, which may affect tumor response and oncologic outcomes [4]. Therefore, predictive biomarkers have been actively investigated for various cancers [5]. Since HCC is typically diagnosed using imaging studies and serum markers, studies using HCC tissue samples are scarce compared with studies on other cancer types. Thus, it is important to find a predictive serum biomarker for patients with HCC. Alpha-fetoprotein (AFP) and protein induced by vitamin $\mathrm{K}$ absence-II (PIVKA-II) are well-known prognostic markers in HCC, and their higher levels correlate with advanced and aggressive disease in many cases [6]. However, AFP and PIVKA-II have limitations in predicting therapeutic results. Previously, our group reported the possibility of using soluble programmed cell death ligand-1 (sPD-L1), plasma cell-free DNA (cfDNA), interleukin (IL)-6, IL-10, and inter-alpha inhibitor H4 (ITIH4) as predictive biomarkers for patients treated with LDCRT [7-10]. However, the optimal single biomarker with substantial predictive power remains unclear. The current study aimed to investigate and incorporate multiple biomarkers in prospectively recruited patients to determine the optimal biomarkers and build an idea for a biomarker-driven treatment paradigm.

\section{Materials and Methods}

\section{Study Population}

This hypothesis-generating study was conducted prospectively and was approved by the institutional review board of the Yonsei University Health System (IRB number: 4-2015-0976, 4-20170093). Patients with HCC who were treated with LDCRT between July 2016 and October 2018 were recruited. All patients were enrolled after obtaining written informed consent. The eligibility criteria included: unresectable primary HCC treated with external beam radiotherapy (RT), over 20 years old, an Eastern Cooperative Oncology Group performance status score of $0-2$, Child-Turcotte-
Pugh's classification A or B, normal liver volume $>1,000 \mathrm{~cm}^{3}$, distance between the liver and organs at risk $\geq 0.5 \mathrm{~cm}$, adequate liver function test (AST/ALT $<5 \times$ upper limit of normal, total bilirubin $<3 \mathrm{mg} / \mathrm{dL}$, albumin $>2.5 \mathrm{~g} / \mathrm{dL}$, normal PT/PTT), adequate renal function (creatinine $<1.8 \mathrm{mg} / \mathrm{dL}$ or Ccr $>50 \mathrm{~mL} / \mathrm{min}$ ), and BM reserve $\left(\mathrm{ANC} \geq 1,500 / \mathrm{mm}^{3}, \mathrm{PLT} \geq 50,000 / \mathrm{mm}^{3}, \mathrm{Hb}>9 \mathrm{~g} / \mathrm{dL}\right)$. Patients who received prior RT to the upper abdomen were excluded.

Unresectable or inoperable HCC patients, whether initially diagnosed or recurred after surgery or local treatment, were discussed in a multidisciplinary tumor board, and the treatment option was decided in accordance with the institution's treatment policy. For patients whose tumor was confined within the liver, liver-directed treatments were administered to reduce tumor burden while preserving systemic therapy for later use. LDCRT was administered as either transarterial chemoembolization plus RT or liver-directed concurrent chemoradiotherapy, which combines local radiotherapy with concurrent hepatic arterial infusion chemotherapy (HAIC) followed by monthly HAIC via chemoport [11]. The rationale of this approach was to maximize local tumor control by a combination of RT and chemotherapy and to minimize intrahepatic metastasis by HAIC [11]. Since the first pilot study in 2008, its efficacy has been documented through several retrospective studies and more recently through a phase 2 clinical trial, which has provided a basis for the institutional policy of applying liver-directed chemoradiotherapy for locally advanced HCC [12-14]. Patients received concurrent HAIC with 5-fluorouracil administered at $500 \mathrm{mg} / \mathrm{m}^{2}$ daily on 5 consecutive days during the first and last weeks of RT. Those who were not accessible for HAIC had oral 5-FU administered as an alternative. As for sorafenib, only 7 patients were prescribed with sorafenib after RT but prior to disease progression (RT cessation to sorafenib prescription: median 87 days [34-134 days]). Since sorafenib was not administered at the beginning of RT but after treatment, it was excluded from analysis due to possible bias.

Since most patients presented with large and/or multiple tumors, conventional RT was administered with a median RT dose of $100 \mathrm{~Gy}$ (range, 60-100 Gy) with a median fractional dose of 4 Gy (2.4-5 Gy) for gross tumor volume (GTV) and a median RT dose of $60 \mathrm{~Gy}$ (range, 45-60 Gy) with a median fractional dose of $2.4 \mathrm{~Gy}(1.8-3 \mathrm{~Gy})$ for planning target volume. GTV was defined as the core of the primary tumor and/or portal vein thrombosis and/ or lymph node. Internal target volume (ITV) was defined as tumor position at each respiratory phase, clinical target volume was defined as ITV plus $5 \mathrm{~mm}$, and planning target volume was defined as CTV plus 5-10 $\mathrm{mm}$. Normal organ constraints concerning liver, stomach, duodenum, and colon were prioritized when planning, while maximizing the dose delivered to the tumor.

\section{Sample Collection}

Blood (5-10 mL) was collected from HCC patients before and after LDCRT in Vacutainer tubes containing EDTA (BD, Franklin Lakes, NJ, USA) using aseptic measures. The window period for the sample collection was 2 weeks for both baseline and post-RT samples. Despite the window period, the baseline sample collection was consistent across the cohort with most sample collected within few days from the start of RT, whereas some post-RT samples were collected more later on after the cessation of RT. The blood samples were centrifuged at 3,000 rpm for $10 \mathrm{~min}$ at $4^{\circ} \mathrm{C}$ to separate the buffy coat and plasma. Additional centrifugation for $10 \mathrm{~min}$ was performed to produce cell-free plasma which was immediately frozen at $-80^{\circ} \mathrm{C}$ until further processing. 
Experimental Techniques

Plasma ITIH4 levels were measured using an ELISA kit according to the manufacturer's protocol (Cloud-Clone Corp., Houston, TX, USA). Absorbance was measured at $450 \mathrm{~nm}$ using an ELISA reader (VERSA Max Microplate Reader; California, USA). ITIH4 levels were calculated based on the generated standard curves [9]. Plasma protein concentrations of the following immune molecules were determined using a magnetic bead-based 6-plex immunoassay: interferon-gamma (IFN- $\gamma$ ), IL-6, IL-10, and sPD-L1 (customized Procartaplex; Thermo Scientific, Waltham, MA, USA). Values are expressed in picograms per millilitre. cfDNA purification and quantification were performed as previously reported [10]. Details of the experimental techniques are included in the online supplementary material (for all online suppl. material, see www. karger.com/doi/10.1159/000522000).

\section{Statistical Analysis}

Baseline biomarkers were the main subject of this hypothesis-generating study, and fold-change values were observed with an exploratory aim. Continuous variables are shown as mean \pm standard deviation. The baseline and post-LDCRT mean biomarker levels were compared using a paired $t$ test. Differences in biomarker levels between patient groups were analyzed using an independent two-sample $t$ test. Clinical outcomes were calculated from the first day of LDCRT to the day of the first event, defined as overall progression for progression-free rate (PFR), progression inside the RT field including progression of initially treated lesion by size and increase in the area of rim enhancement for local failure-free rate (LFFR), progression within the liver but outside the RT field for intrahepatic failure-free rate (IHFFR), and lung metastasis for lung metastasis-free rate (LMFR). Local failure was determined using the modified response evaluation criteria for solid tumors. The cumulative probabilities of freedom from progression, local failure, and intrahepatic failure lung metastasis were calculated using Kaplan-Meier method and compared using a logrank test. Univariate and multivariate analyses were performed using Cox regression with a backward stepwise method. All variables were initially included, and unfit variables which did not contribute to the regression equation were excluded using a backward stepwise method. Variables that remained at the final stage are included in the tables. $p$ values (in two-sided tests) $\leq 0.05$ were considered statistically significant. Survival tree analysis including all continuous baseline biomarker values and clinical variables was used to determine prognostic groups. For baseline biomarker analysis, all six biomarkers were included. However, for fold-change values, IFN- $\gamma$ was excluded from the analysis, as many patients had a baseline value of zero. Data were analyzed using IBM SPSS software (version 23.0; SPSS Inc., Chicago, IL, USA), R version 3.6.1. (R Foundation for Statistical Computing, Vienna, Austria), and GraphPad Prism (GraphPad, San Diego, CA, USA).

\section{Results}

\section{Patient and Tumor Characteristics}

A total of 102 patients were prospectively enrolled in this study. Three patients who did not undergo follow-up imaging studies were excluded, leaving 99 patients in the
Table 1. Patient, tumor, and treatment characteristics $(n=99)$

\begin{tabular}{|c|c|c|}
\hline & $n$ & $\%$ \\
\hline Age, years (range) & $61(33-80)$ & \\
\hline \multicolumn{3}{|l|}{ Sex } \\
\hline Male & 78 & 78.8 \\
\hline Female & 21 & 21.2 \\
\hline \multicolumn{3}{|l|}{ Viral type } \\
\hline B-viral & 72 & 72.7 \\
\hline C-viral & 6 & 6.1 \\
\hline Non- $B$, non- $C$ viral & 21 & 21.2 \\
\hline \multicolumn{3}{|l|}{ Child-Pugh class } \\
\hline A & 87 & 87.9 \\
\hline B & 12 & 12.1 \\
\hline \multicolumn{3}{|l|}{ Liver cirrhosis } \\
\hline No & 54 & 54.5 \\
\hline Yes & 45 & 45.5 \\
\hline Tumor size, cm (range) & $6.5(1.3-21.0)$ & \\
\hline \multicolumn{3}{|l|}{ Tumor, $n$} \\
\hline One & 44 & 44.4 \\
\hline Multiple & 55 & 55.6 \\
\hline \multicolumn{3}{|l|}{ UICC stage } \\
\hline Stage II & 27 & 27.3 \\
\hline Stage III & 32 & 32.3 \\
\hline Stage IV & 40 & 40.4 \\
\hline \multicolumn{3}{|l|}{ BCLC stage } \\
\hline Stage A & 24 & 24.2 \\
\hline Stage B & 26 & 26.3 \\
\hline Stage C & 49 & 49.5 \\
\hline \multicolumn{3}{|l|}{ Portal vein thrombosis } \\
\hline No & 52 & 52.5 \\
\hline Yes & 47 & 47.5 \\
\hline AFP, ng/mL (median, range) & $71.13(1.68-268,407.8)$ & \\
\hline PIVKA-II, mAU/mL (median, range) & $1,629.0(15.0-75,000.0)$ & \\
\hline \multicolumn{3}{|l|}{ Treatment aim } \\
\hline Definitive & 58 & 58.6 \\
\hline Salvage & 41 & 41.4 \\
\hline \multicolumn{3}{|l|}{ Previous treatment } \\
\hline No & 51 & 51.5 \\
\hline Yes & 48 & 48.5 \\
\hline \multicolumn{3}{|l|}{ Concurrent chemotherapy } \\
\hline Concurrent chemoradiotherapy & 66 & 66.7 \\
\hline HAIC (5-fluorouracil) & 63 & 63.6 \\
\hline Tegafur/uracil & 3 & 3.0 \\
\hline Radiotherapy alone & 33 & 33.3 \\
\hline \multicolumn{3}{|l|}{ Radiotherapy dose to PTV } \\
\hline Total dose, Gy (range) & $60(45-60)$ & \\
\hline Fractional dose, Gy (range) & $2.4(1.8-3.0)$ & \\
\hline
\end{tabular}

UICC, Union for International Cancer Control; BCLC, Barcelona clinic liver cancer; PTV, planning target volume.

analysis. The median follow-up period was 18.7 months (2.9-34.8 months). Patient and tumor characteristics are shown in Table 1 . The median age was 61 years $(33-80$ years), and majority of patients were men. Most patients were diagnosed with B-viral type HCC and were ChildPugh class A; approximately half of the patients had liver 


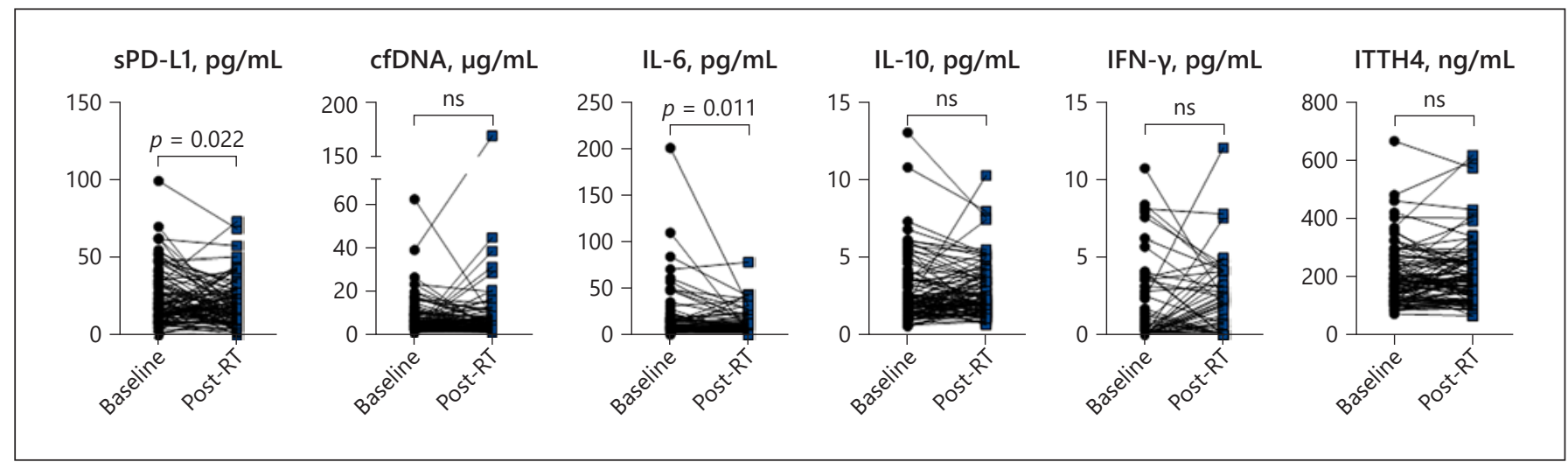

Fig. 1. Individual changes showing baseline and post-radiotherapy biomarker values.

cirrhosis at the time of LDCRT. Forty-four patients (44.4\%) had a single tumor, and the median tumor size was $6.5 \mathrm{~cm}(1.3-21.0 \mathrm{~cm})$. Nine patients had node-positive disease, 52 had portal vein tumor thrombosis or invasion, and 40 had UICC stage IV disease. Treatment characteristics are listed in Table 1.

The mean baseline level for the biomarkers were sPD-L1: $23.94 \pm 17.66 \mathrm{pg} / \mathrm{mL}$ (1.95-98.76 pg/mL); IFN- $\gamma$ : $0.99 \pm 2.19 \mathrm{pg} / \mathrm{mL}(0-10.70 \mathrm{pg} / \mathrm{mL}) ; \mathrm{IL}-6: 15.46 \pm 26.26$ $\mathrm{pg} / \mathrm{mL}(0.10-199.85 \mathrm{pg} / \mathrm{mL}) ; \mathrm{IL}-10: 2.73 \pm 2.06 \mathrm{pg} / \mathrm{mL}$ (0.57-12.99 pg/mL); ITIH4: $197.91 \pm 99.48 \mathrm{ng} / \mathrm{mL}(72.30-$ $664.20 \mathrm{ng} / \mathrm{mL})$; and cfDNA: $7.35 \pm 7.91 \mu \mathrm{g} / \mathrm{mL}(1.00$ $59.12 \mu \mathrm{g} / \mathrm{mL}$ ). Mean IL-6 levels were higher in node-positive than in node-negative patients $(42.51 \%$ vs. $12.76 \%$, $p=0.001)$. Patients with a tumor size larger than $6.5 \mathrm{~cm}$ showed higher mean IL-6 $(22.14 \mathrm{pg} / \mathrm{mL}$ vs. $9.18 \mathrm{pg} / \mathrm{mL}$, $p=0.016)$ and higher IL-10 $(3.38 \mathrm{pg} / \mathrm{mL}$ vs. $2.12 \mathrm{pg} / \mathrm{mL}$, $p=0.003)$ levels. Individual changes in the baseline and post-LDCRT levels are shown in Figure 1.

\section{Clinical Outcomes}

At 1-month follow-up after LDCRT, complete response, partial response, stable disease (SD), and progressive disease were observed in $2(2.0 \%), 65(65.7 \%), 29$ (29.3\%), and 3 (3.0\%) patients, respectively. Sixty-six patients eventually showed disease progression. The first failure patterns are shown in online supplementary Figure 1. The most common first pattern of failure was regional-only failure, including intrahepatic failure and regional lymph node metastasis, followed by distant-only failure. The lung was the most common site of distant failure. Lung metastases accounted for $73 \%$ (32 of 44) of all distant failure events. Other sites of distant failure in- cluded bones, peritoneal seeding, supraclavicular lymph node, abdominal wall, and adrenal gland. The 1-year PFR was $38.2 \%$, and the median time to progression was 5.0 months (0.8-24.8 months). The 1-year LFFR, IHFFR, and LMFR were $85.7 \%, 50.7 \%$, and $76.0 \%$, respectively.

\section{Significance of Baseline Levels}

Multivariate analysis was performed, including the baseline biomarker levels. For disease progression, multiple tumors (hazard ratio [HR]: 2.00; 95\% confidence interval [CI]: 1.24-3.23; $p=0.005$ ), previous treatment history (HR: 1.99; CI: $1.16-3.42 ; p=0.013)$, PIVKA-II $>1,629 \mathrm{mAU} / \mathrm{mL}$ (HR: 1.79 ; CI: $1.04-3.09 ; p=0.036$ ), and increasing baseline sPD-L1 per pg/mL (HR: 1.02; CI: $1.00-1.03 ; p=0.041$ ) were significant adverse factors (Table 2). For local failure, the presence of liver cirrhosis (HR: 5.74; CI: $1.48-18.54 ; p=$ 0.010 ), and PIVKA-II >1,629 mAU/mL (HR: 4.65; CI: $1.11-$ $19.42 ; p=0.035)$ were significant adverse factors. For intrahepatic failure, multiple tumors (HR: 2.93; CI: 1.63-5.26; $p<0.001$ ), not receiving concurrent chemotherapy (HR: 3.18; CI: $1.67-6.05 ; p<0.001)$ and increasing baseline sPD-L1 per pg/mL (HR: 1.01 ; CI: $1.00-1.03 ; p=0.043$ ) were unfavorable factors. Adjusting for other factors, increasing baseline SPD-L1 was found to be a significant adverse factor for lung metastasis (HR: 1.02; CI: $1.00-1.04 ; p=0.042$ ), whereas not receiving concurrent chemotherapy was a favorable factor (HR: 0.16; CI: $0.05-0.54 ; p=0.003$ ) (online suppl. Table S1). For overall distant metastasis, receiving concurrent chemotherapy (HR: 0.33; CI: $0.13-0.88$; $p=$ $0.026)$, receiving previous treatment (HR: 3.14 ; CI: 14.59$6.78 ; p=0.003$ ) and higher UICC stage (II vs. III; HR: 3.88; CI: $1.67-11.00 ; p=0.011$, II vs. IV; HR: 3.62, CI: 1.23-10.67, $p=0.019)$ were significant factors. 
Table 2. Analysis of factors associated with disease progression including baseline biomarker levels

\begin{tabular}{|c|c|c|c|c|c|c|}
\hline \multirow[t]{2}{*}{ Variable } & \multicolumn{3}{|l|}{ UVA } & \multicolumn{3}{|l|}{ MVA } \\
\hline & $\mathrm{HR}$ & $95 \% \mathrm{Cl}$ & $p$ value & $\mathrm{HR}$ & $95 \% \mathrm{Cl}$ & $p$ value \\
\hline Sex (female vs. male) & 0.94 & $0.55-1.62$ & 0.832 & & & \\
\hline Tumor number (multiple vs. one) & 1.91 & $1.19-3.08$ & 0.007 & 2.00 & $1.24-3.23$ & 0.005 \\
\hline Liver cirrhosis (yes vs. no) & 1.00 & $0.63-1.58$ & 0.989 & & & \\
\hline Child-pugh class (B vs. A) & 1.40 & $0.70-2.83$ & 0.345 & & & \\
\hline Concurrent chemotherapy (no vs. yes) & 0.99 & $0.62-1.62$ & 0.989 & & & \\
\hline PVTT (yes vs. no) & 1.33 & $0.84-2.10$ & 0.225 & & & \\
\hline Previous treatment (yes vs. no) & 1.34 & $0.85-2.12$ & 0.207 & 1.99 & $1.16-3.42$ & 0.013 \\
\hline Age $(>60$ vs. $\leq 60)$ & 1.01 & $0.64-1.60$ & 0.953 & & & \\
\hline Tumor size (>6.5 cm vs. $\leq 6.5 \mathrm{~cm})$ & 1.01 & $0.64-1.60$ & 0.964 & & & \\
\hline Lymph node metastasis (yes vs. no) & 0.95 & $0.41-2.19$ & 0.896 & & & \\
\hline Treatment aim (salvage vs. definitive) & 1.15 & $0.73-1.82$ & 0.551 & & & \\
\hline UICC stage & & & 0.088 & & & \\
\hline III versus II & 1.66 & $0.91-3.04$ & 0.100 & & & \\
\hline IV versus II & 1.92 & $1.07-3.46$ & 0.030 & & & \\
\hline $\operatorname{AFP}(>71.13 \mathrm{ng} / \mathrm{mL}$ vs. $\leq 71.13 \mathrm{ng} / \mathrm{mL})$ & 1.41 & $0.89-2.22$ & 0.146 & & & \\
\hline PIVKA-II (>1,629 mAU/mL vs. $\leq 1,629 \mathrm{mAU} / \mathrm{mL})$ & 1.35 & $0.85-2.13$ & 0.207 & 1.79 & $1.04-3.09$ & 0.036 \\
\hline $\mathrm{sPD}-\mathrm{L} 1(\mathrm{pg} / \mathrm{mL})$ & 1.01 & $1.00-1.03$ & 0.044 & 1.02 & $1.00-1.03$ & 0.041 \\
\hline $\mathrm{IFN}-\gamma(\mathrm{pg} / \mathrm{mL})$ & 1.00 & $0.90-1.11$ & 0.991 & & & \\
\hline IL-6 (pg/mL) & 1.00 & $0.99-1.01$ & 0.744 & & & \\
\hline $\mathrm{IL}-10(\mathrm{pg} / \mathrm{mL})$ & 1.08 & $0.96-1.22$ & 0.189 & & & \\
\hline ITIH4 (ng/mL) & 1.00 & $1.00-1.00$ & 0.194 & & & \\
\hline cfDNA $(\mu \mathrm{g} / \mathrm{mL})$ & 1.03 & $1.00-1.06$ & 0.074 & & & \\
\hline
\end{tabular}

PVTT, portal vein tumor thrombosis; UICC, Union for International Cancer Control.

Survival tree analysis was used to determine the prognostic groups for disease progression, including all clinical factors and biomarkers. Three prognostic groups were identified for progression based on SPD-L1 and the number of hepatic lesions: group 1, single lesion; group 2, multiple lesions and sPD-L1 $<41.07 \mathrm{pg} / \mathrm{mL}$; and group 3, multiple lesions and sPD-L1 $\geq 41.07 \mathrm{pg} / \mathrm{mL}$ (online suppl. Fig. $2)$. Overall progression was highest in group 3 and lowest in group $1(p<0.001$, shown in Fig. 2$)$.

\section{Significance of Fold-Change Values}

Multivariate analysis was performed for clinical outcomes, including fold-change values. For disease progression, receiving previous treatment (HR: 3.14; CI: 1.715.79; $p<0.001$ ), age $>60$ years (HR: 0.37 ; CI: $0.15-0.90$; $p=0.029$ ), UICC stage IV (HR: 3.47 ; CI: $1.73-6.98 ; p<$ 0.001 ), and stage III (HR: 2.72; CI: $1.40-5.30 ; p=0.003$ ) compared with stage II, higher fold increase of sPD-L1 (HR: 1.02; CI: $1.00-1.03 ; p=0.023$ ), and a higher fold increase of cfDNA (HR: 1.12 ; CI: $1.01-1.24 ; p=0.029$ ) were significant adverse factors (Table 3). Furthermore, a higher fold increase in cfDNA was found to be an adverse factor for both local failure (HR: 1.22; CI: 1.08-1.39; $p=$ 0.001 ) and intrahepatic failure (HR: 1.20; CI: 1.08-1.33; $p<0.001$ ) (online suppl. Table S2).

\section{Discussion}

We investigated the clinical significance of six molecules (sPD-L1, IL-10, IL-6, cfDNA, ITIH4, and IFN- $\gamma$ ) as biomarkers for HCC patients treated with LDCRT. Baseline sPD-L1 appeared to be the most useful biomarker, with increased baseline levels significantly associated with worse PFR, IHFFR, and LMFR. Furthermore, prognostic subgroups were identified by incorporating both clinical characteristics and biomarkers. A higher fold increase in sPD-L1 expression was associated with increased overall progression. Additionally, a higher fold increase in cfDNA was related to worse PFR, LFFR, and IHFFR.

Many biomarkers have been reported as potential predictors in cancer patients; IL-10 has dual effects of both immune suppression and immune stimulation [15]. In- 
Fig. 2.Kaplan-Meier estimates of overall progression for three groups determined by survival tree analysis.

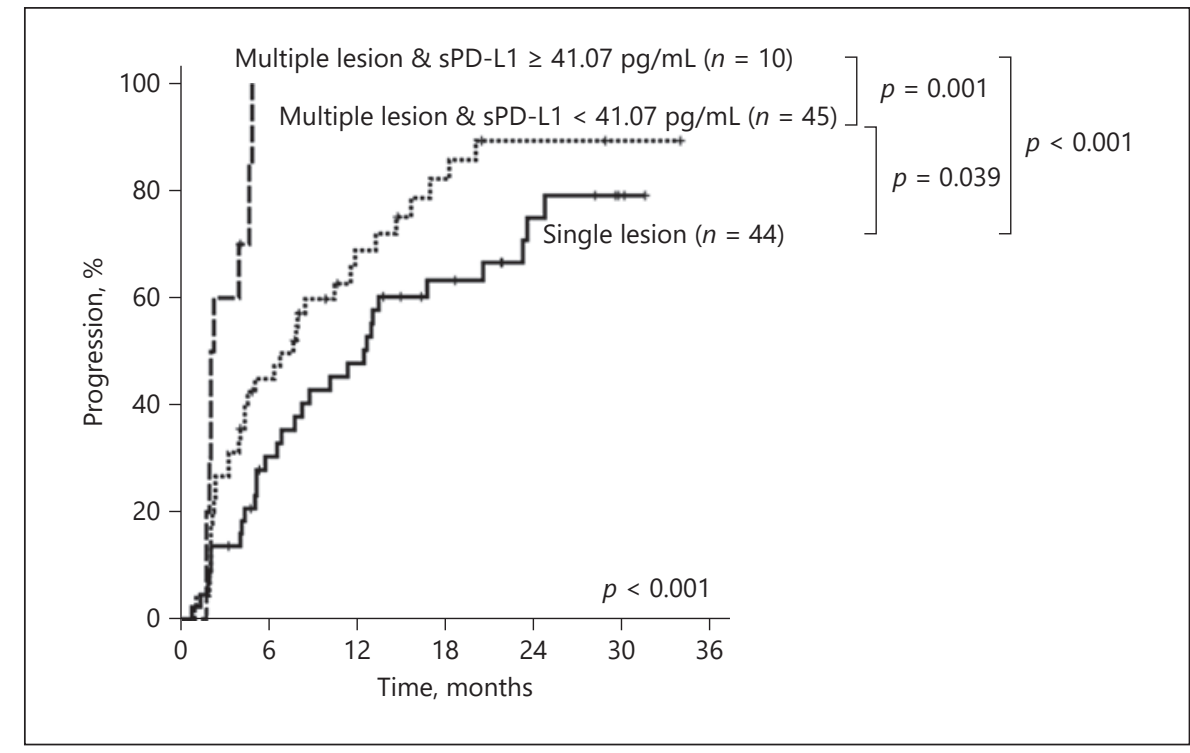

Table 3. Analysis of factors associated with disease progression including biomarker fold-change values

\begin{tabular}{|c|c|c|c|c|c|c|}
\hline \multirow[t]{2}{*}{ Variable } & \multicolumn{3}{|l|}{ UVA } & \multicolumn{3}{|l|}{ MVA } \\
\hline & $\mathrm{HR}$ & $95 \% \mathrm{Cl}$ & $p$ value & $\mathrm{HR}$ & $95 \% \mathrm{Cl}$ & $p$ value \\
\hline Sex (female vs. male) & 0.94 & $0.55-1.62$ & 0.832 & & & \\
\hline Tumor number (multiple vs. one) & 1.91 & $1.19-3.08$ & 0.007 & & & \\
\hline Liver cirrhosis (yes vs. no) & 1.00 & $0.63-1.58$ & 0.989 & & & \\
\hline Child-pugh class (B vs. A) & 1.40 & $0.70-2.83$ & 0.345 & & & \\
\hline Concurrent chemotherapy (no vs. yes) & 0.99 & $0.62-1.62$ & 0.989 & & & \\
\hline PVTT (yes vs. no) & 1.33 & $0.84-2.10$ & 0.225 & & & \\
\hline Previous treatment (yes vs. no) & 1.34 & $0.85-2.12$ & 0.207 & 3.14 & $1.71-5.79$ & $<0.001$ \\
\hline Age (>60 vs. $\leq 60)$ & 1.01 & $0.64-1.60$ & 0.953 & 0.37 & $0.15-0.90$ & 0.029 \\
\hline Tumor size (>6.5 cm vs. $\leq 6.5 \mathrm{~cm})$ & 1.01 & $0.64-1.60$ & 0.964 & & & \\
\hline Lymph node metastasis (yes vs. no) & 0.95 & $0.41-2.19$ & 0.896 & & & \\
\hline Treatment aim (salvage vs. definitive) & 1.15 & $0.73-1.82$ & 0.551 & & & \\
\hline UICC stage & & & 0.088 & & & 0.001 \\
\hline III versus II & 1.66 & $0.91-3.04$ & 0.100 & 2.72 & $1.40-5.30$ & 0.003 \\
\hline IV versus II & 1.92 & $1.07-3.46$ & 0.030 & 3.47 & $1.73-6.98$ & $<0.001$ \\
\hline $\operatorname{AFP}(>71.13 \mathrm{ng} / \mathrm{mL}$ vs. $\leq 71.13 \mathrm{ng} / \mathrm{mL})$ & 1.41 & $0.89-2.22$ & 0.146 & & & \\
\hline PIVKA-II (>1,629 mAU/mL vs. $\leq 1,629 \mathrm{mAU} / \mathrm{mL})$ & 1.35 & $0.85-2.13$ & 0.207 & 1.76 & $0.99-3.09$ & 0.052 \\
\hline sPD-L1 fold change & 1.01 & $1.00-1.03$ & 0.062 & 1.02 & $1.00-1.03$ & 0.023 \\
\hline IL- 6 fold change & 0.98 & $0.94-1.03$ & 0.460 & & & \\
\hline IL-10 fold change & 1.07 & $0.82-1.38$ & 0.622 & & & \\
\hline ITIH4 fold change & 1.09 & $0.89-1.72$ & 0.721 & & & \\
\hline cfDNA fold change & 1.07 & $0.99-1.17$ & 0.106 & 1.12 & $1.01-1.24$ & 0.029 \\
\hline
\end{tabular}

PVTT, portal vein tumor thrombosis; UICC, Union for International Cancer Control.

creased IL-10 levels were previously associated with poor clinical outcomes in breast, thyroid, and oral cancer tissues $[16,17]$. In the current study, although higher mean IL-10 level was associated with tumor size larger than 6.5 $\mathrm{cm}$, it was not associated with clinical outcomes. This may be due to the fact that IL-10 shows contradictory effects related to tumor growth or tumor regression and that the balance of cytokines and other environmental conditions control its function in different circumstances $[18,19]$. Thus, IL-10 may not be the optimal biomarker in cases of 
locally advanced HCC patients receiving LDCRT. As for IL-6, patients with high IL-6 levels showed worse infield failure-free survival in a previous report [7]. Moreover, in esophageal cancer, higher IL-6 levels were associated with advanced tumor stage, nodal metastasis, and poorer survival [20]. Along with previous studies, our current study indicates that increased baseline IL-6 levels are associated with larger tumor size and nodal metastasis. However, studies on the predictive use of IL- 6 are very scarce, especially in HCC patients [21]. In our study, IL-6 level did not show significant results regarding clinical outcomes. Further studies are needed to confirm the predictive role of IL-6 in HCC patients. Serum ITIH4 was recently associated with malignancies, and in some reports, lower pretreatment serum ITIH4 levels were associated with shorter survival and progression in HCC patients [22]. However, the significance of serum ITIH4 is contradictory as other study reported the relation of higher serum ITIH4 levels correlating with poorer prognosis [23]. The current study shows that other biomarkers such as SPD-L1 or cfDNA rather than ITIH4 may have more potential to be a significant biomarker in HCC patients receiving LDCRT. IFN- $\gamma$ shows anti- and pro-tumorigenic activities depending on the cellular, microenvironmental, and molecular settings [24]. Subsequently, studies report variable results on the role of IFN- $\gamma$ level in cancer patients [25, 26]. In this study, IFN- $\gamma$ did not reveal as a significant biomarker, and it seems that further research efforts are necessary to decipher the IFN- $\gamma$-related effects in cancer patients. In addition, previously well-known prognostic markers such as AFP and PIVKA-II were not significant factors in this study. The fact that this study cohort mostly included locally advanced HCC patients may have affected the results. Since the well-known prognostic markers reflect the intrahepatic burden, its efficacy may be decreased in a homogenous cohort mostly including patients with a large tumor burden. Thus, this may show the limitation of AFP and PIVKA-II in these locally advanced HCC patients who received LDCRT. Overall, the significant biomarkers compared favorably to AFP or PIVKA-II in this cohort.

Immune checkpoint inhibitors have gained immense attention. In HCC, a phase III study recently showed significant improvement in survival and progression-free survival for a combination of atezolizumab and bevacizumab compared with sorafenib in patients with unresectable HCC without previous systemic therapy [27]. One of the most actively used types of immune checkpoint inhibitors is programmed death ligand-1 (PD-L1) inhibitors. PD-L1 expression is associated with poor

Biomarkers for Locally Advanced Hepatocellular Carcinoma Patients prognosis in various types of cancers [28]. PD-L1 expression can be detected by either immunohistochemistry or in blood samples by enzyme-linked immunosorbent assay, as $\mathrm{SPD}-\mathrm{L} 1$ is released from PD-L1-positive cells into the serum. We have previously reported that high sPD-L1 is associated with worse OS in patients with HCC [9]. Thus, sPD-L1 could be a potential biomarker for patients with HCC. However, the optimal single biomarker with substantial predictive power remains unclear. In the current study, among the six biomarkers, baseline sPD-L1 was found to be the best, showing a strong correlation with HCC progression, intrahepatic failure, and lung metastasis. However, clinical characteristics should be considered along with biomarker levels when predicting patient prognosis. In the current study, all clinical characteristics and biomarkers were included in the analysis to find three prognostic groups, with the group of patients with multiple lesions and sPD-L1 $\geq 41.07 \mathrm{pg} / \mathrm{mL}$ showing the worst results. Furthermore, when considering further systemic treatments, such as immunotherapy, in these sPD-L1 high HCC patients, an additional PD-L1 antibody may be required for PD-L1-based immunotherapy, as SPD-L1 binds to anti-PD-L1 antibody.

cfDNA is a potential surrogate marker for many indications in cancer patients, including response to treatment [29]. Previous studies showed correlation between cfDNA levels and treatment response in other cancer types, in which higher baseline cfDNA levels were related to poor response to treatment [30-32]. The level of cfDNA is influenced by multiple factors, including a wide range of biological and environmental factors. It originates from tumor cells and other cells in the tumor microenvironment [33]. Thus, whereas cfDNA from tumor cells alone, which can be called circulating tumor DNA, is important for diagnosing cancer, the measurement of the levels of total cfDNA and the calculation of its fold change may be more expedient for predicting treatment response, considering factors such as tumor dynamics and changes in tumor microenvironment.

Considering the factors discussed thus far, the idea of biomarker-driven treatment strategies that might be applied to patients with locally advanced HCC can be hypothesized. For example, locally advanced HCC patients with single or multiple lesions who show baseline sPD-L1 levels lower than $41.07 \mathrm{pg} / \mathrm{mL}$ may benefit from LDCRT. However, patients with multiple tumors and baseline sPD-L1 levels higher than $41.07 \mathrm{pg} / \mathrm{mL}$ may need stronger systemic treatment along with LDCRT. In particular, a more effective treatment strategy may be necessary for patients with a higher fold increase in SPD-L1 and cfD- 
NA. In addition, in line with the suggested main treatment strategy, patients with high baseline sPD-L1 levels at risk of intrahepatic failure and lung metastasis may need stronger systemic treatment along with LDCRT. After treatment, those who show higher fold increase in sPD-L1 and cfDNA will also need to consider active surveillance with appropriate imaging modality.

In this study, patients received conventionally fractionated RT as part of LDCRT. Considering our previous reports showing different patterns of sPD-L1 change by different RT schemes in fractionation [8], this remains a limitation of this study. Further studies on patients receiving different RT schemes are currently underway. Also, a larger number of patients are needed to fully elucidate powerful biomarkers. Nonetheless, the results of this hypothesis-generating study may provide further insights into improving the oncologic outcome of locally advanced HCC.

In conclusion, baseline sPD-L1 and cfDNA foldchange values showed the greatest potential as predictive biomarkers for HCC patients who received LDCRT. By incorporating clinical factors, these biomarkers may be useful for devising a biomarker-driven treatment paradigm in locally advanced HCC. Further validation studies are required.

\section{Statement of Ethics}

This hypothesis-generating study was conducted prospectively and was approved by the institutional review board of the Yonsei University Health System (IRB number: 4-2015-0976, 4-2017-0093). Written informed consent was obtained from participants (or their parent/legal guardian/next of kin) to participate in the study.

\section{Conflict of Interest Statement}

The authors have no conflicts of interest to declare.

\section{Funding Sources}

This study was supported by the National Nuclear R\&D Program through the National Research Foundation of Korea (NRF) funded by the Ministry of Science and ICT (Grant No. 2017070426) and by the Dong-A research fund (Grant No. 2018-31-0904). The sponsors did not have a role in any of the stages from study design to submission of the paper for publication.

\section{Author Contributions}

All authors contributed to the study conception and design. Material preparation, data collection, and analysis were performed by Seung Yeun Chung, Kyoung-Jin Kim, and Jinsil Seong. The first draft of the manuscript was written by Seung Yeun Chung, and all authors critically reviewed previous versions of the manuscript. All authors read and approved the final manuscript and have agreed on the journal to which the article will be submitted. All authors agreed to take responsibility and be accountable for the contents of the article.

\section{Data Availability Statement}

All data generated or analyzed during this study are included in this article and its online supplementary Files. Further enquiries can be directed to the corresponding author.

\section{References}

1 European Association for the Study of the Liver; European Organisation for Research and Treatment of Cancer. EASL-EORTC clinical practice guidelines: management of hepatocellular carcinoma. J Hepatol. 2012 Apr;56(4):908-43.

2 Forner A, Reig ME, de Lope CR, Bruix J. Current strategy for staging and treatment: the BCLC update and future prospects. Semin Liver Dis. 2010 Feb;30(1):61-74.

3 Lee WH, Byun HK, Choi JS, Choi GH, Han $\mathrm{DH}$, Joo DJ, et al. Liver-directed combined radiotherapy as a bridge to curative surgery in locally advanced hepatocellular carcinoma beyond the Milan criteria. Radiother Oncol. 2020 Nov; 152:1-7.
4 Gonzalez H, Hagerling C, Werb Z. Roles of the immune system in cancer: from tumor initiation to metastatic progression. Genes Dev. 2018;32(19-20):1267-84.

5 Ludwig JA, Weinstein JN. Biomarkers in cancer staging, prognosis and treatment selection. Nat Rev Cancer. 2005;5(11):845.

6 Suehiro T, Sugimachi K, Matsumata T, Itasaka H, Taketomi A, Maeda T. Protein induced by vitamin $\mathrm{K}$ absence or antagonist II as a prognostic marker in hepatocellular carcinoma. Comparison with alpha-fetoprotein. Cancer. 1994;73(10):2464-71.

7 Cha H, Lee EJ, Seong J. Multi-analyte analysis of cytokines that predict outcomes in patients with hepatocellular carcinoma treated with radiotherapy. World J Gastroenterol. 2017 Mar 21;23(11):2077-85.
8 Kim HJ, Park S, Kim KJ, Seong J. Clinical significance of soluble programmed cell death ligand-1 (sPD-L1) in hepatocellular carcinoma patients treated with radiotherapy. Radiother Oncol. 2018 Oct;129(1):130-5.

9 Lee EJ, Yang SH, Kim KJ, Cha H, Lee SJ, Kim $\mathrm{JH}$, et al. Inter-alpha inhibitor $\mathrm{H} 4$ as a potential biomarker predicting the treatment outcomes in patients with hepatocellular carcinoma. Cancer Res Treat. 2018 Jul;50(3):64657.

10 Park S, Lee EJ, Rim CH, Seong J. Plasma cellfree DNA as a predictive marker after radiotherapy for hepatocellular carcinoma. Yonsei Med J. 2018 Jun;59(4):470-9. 
11 Han KH, Seong J, Kim JK, Ahn SH, Lee DY, Chon CY. Pilot clinical trial of localized concurrent chemoradiation therapy for locally advanced hepatocellular carcinoma with portal vein thrombosis. Cancer. 2008;113(5): 995-1003.

12 Lee HS, Choi GH, Choi JS, Kim KS, Han K-H, Seong J, et al. Surgical resection after down-staging of locally advanced hepatocellular carcinoma by localized concurrent chemoradiotherapy. Ann Surg Oncol. 2014; 21(11):3646-53.

13 Chong JU, Choi GH, Han DH, Kim KS, Seong $\mathrm{J}$, Han KH, et al. Downstaging with localized concurrent chemoradiotherapy can identify optimal surgical candidates in hepatocellular carcinoma with portal vein tumor thrombus. Ann Surg Oncol. 2018;25(11):3308-15.

14 Kim BK, Byun HK, Choi HJ, Beom S-H, Lee HW, Kim SU, et al. Efficacy and safety of liver-directed concurrent chemoradiotherapy and sequential sorafenib for advanced hepatocellular carcinoma; a prospective phase 2 trial. Int J Radiat Oncol Biol Phys. 2020; 107(1):106-15.

15 Mocellin S, Panelli MC, Wang E, Nagorsen D, Marincola FM. The dual role of IL-10. Trends Immunol. 2003;24(1):36-43.

16 Wang S, Sun M, Gu C, Wang X, Chen D, Zhao E, et al. Expression of CD 163, interleukin-10, and interferon-gamma in oral squamous cell carcinoma: mutual relationships and prognostic implications. Eur J Oral Sci. 2014; 122(3):202-9.

17 Cunha LL, Morari EC, Nonogaki S, Marcello MA, Soares FA, Vassallo J, et al. Interleukin 10 expression is related to aggressiveness and poor prognosis of patients with thyroid cancer. Cancer Immunol Immunother. 2017; 66(2):141-8.
18 Mannino MH, Zhu Z, Xiao H, Bai Q, Wakefield MR, Fang Y. The paradoxical role of IL10 in immunity and cancer. Cancer Lett. 2015; 367(2):103-7.

19 Berti FCB, de Oliveira KB. IL-10 in cancer: just a classical immunosuppressive factor or also an immunostimulating one? AIMS Allergy Immunol. 2018;2(2):88-97.

20 Chen MF, Chen PT, Lu MS, Lin PY, Chen WC, Lee KD. IL-6 expression predicts treatment response and outcome in squamous cell carcinoma of the esophagus. Mol Cancer. 2013 Apr 5;12:26.

21 Vainer N, Dehlendorff C, Johansen JS. Systematic literature review of IL- 6 as a biomarker or treatment target in patients with gastric, bile duct, pancreatic and colorectal cancer. Oncotarget. 2018;9(51):29820.

22 Noh CK, Kim SS, Kim DK, Lee HY, Cho HJ, Yoon SY, et al. Inter-alpha-trypsin inhibitor heavy chain $\mathrm{H} 4$ as a diagnostic and prognostic indicator in patients with hepatitis B virus-associated hepatocellular carcinoma. Clin Biochem. 2014 Sep;47(13-14):1257-61.

23 Nakamura N, Hatano E, Iguchi K, Sato M, Kawaguchi H, Ohtsu I, et al. Elevated levels of circulating ITIH4 are associated with hepatocellular carcinoma with nonalcoholic fatty liver disease: from pig model to human study. BMC Cancer. 2019;19(1):1-14.

24 Zaidi MR, Merlino G. The two faces of interferon- $\gamma$ in cancer. Clin Cancer Res. 2011 Oct 1;17(19):6118-24.

25 Lee IC, Huang YH, Chau GY, Huo TI, Su CW, $\mathrm{Wu}$ JC, et al. Serum interferon gamma level predicts recurrence in hepatocellular carcinoma patients after curative treatments. Int J Cancer. 2013;133(12):2895-902.
26 Sánchez-Zauco N, Torres J, Gómez A, Camorlinga-Ponce M, Muñoz-Pérez L, Herrera-Goepfert $\mathrm{R}$, et al. Circulating blood levels of IL-6, IFN- $\gamma$, and IL-10 as potential diagnostic biomarkers in gastric cancer: a controlled study. BMC Cancer. 2017;17(1):1-10.

27 Cheng A-L, Qin S, Ikeda M, Galle P, Ducreux $\mathrm{M}$, Zhu A, et al. IMbrave150: efficacy and safety results from a ph III study evaluating atezolizumab (atezo)+ bevacizumab (bev) vs sorafenib (Sor) as first treatment (tx) for patients (pts) with unresectable hepatocellular carcinoma (HCC). Ann Oncol. 2019;30: IX186-7.

28 Wu P, Wu D, Li L, Chai Y, Huang J. PD-L1 and survival in solid tumors: a meta-analysis. PLoS One. 2015;10(6):e0131403.

29 Bronkhorst AJ, Ungerer V, Holdenrieder S. The emerging role of cell-free DNA as a molecular marker for cancer management. Biomol Detect Quantif. 2019 Mar; 17:100087.

30 Tie J, Kinde I, Wang Y, Wong HL, Roebert J, Christie M, et al. Circulating tumor DNA as an early marker of therapeutic response in patients with metastatic colorectal cancer. Ann Oncol. 2015 Aug;26(8):1715-22.

31 Cabanero M, Tsao MS. Circulating tumour DNA in EGFR-mutant non-small-cell lung cancer. Curr Oncol. 2018 Jun;25(Suppl 1): S38-44.

32 O'Leary B, Hrebien S, Morden JP, Beaney M, Fribbens C, Huang X, et al. Early circulating tumor DNA dynamics and clonal selection with palbociclib and fulvestrant for breast cancer. Nat Commun. 2018 Mar 1;9(1):896.

33 Thierry AR, El Messaoudi S, Gahan PB, Anker P, Stroun M. Origins, structures, and functions of circulating DNA in oncology. Cancer Metastasis Rev. 2016 Sep;35(3):347-76. 